\title{
Spatially Inhomogeneous Superconducting and Bosonic Networks with Emergent Complex Behaviors
}

\author{
F. P. Mancini ${ }^{1}$, P. Sodano ${ }^{1}$ and A. Trombettoni ${ }^{2}$ \\ ${ }^{1}$ Dipartimento di Fisica and Sezione I.N.F.N., \\ Università di Perugia, Via A. Pascoli, Perugia, I-06123, Italy and \\ 2 S.I.S.S.A. and Sezione I.N.F.N., Via Beirut 2/4, I-34014 Trieste, Italy
}

(Dated: November 2, 2018)

\begin{abstract}
The spontaneous emergence of enhanced responses and local orders are properties often associated with complex matter where nonlinearities and spatial inhomogeneities dominate. We discuss these phenomena in quantum devices realized with superconducting Josephson junction networks and cold atoms in optical lattices. We evidence how the pertinent engineering of the network's shape induces the enhancement of the zero-voltage Josephson critical currents in superconducting arrays as well as the emergence of spatially localized condensates for cold atoms in inhomogeneous optical lattices.
\end{abstract}

DFUPG: $39-07$ 


\section{INTRODUCTION}

In all fields of physics homogeneous systems have the simplest properties and, thus, play a very important paradigmatic role in our understanding of natural phenomena. It is a fact, however, that many real systems are inhomogeneous in a way or another and that, in some instances, their inhomogeneity may be the seed for the emergence of new and unexpected complex behaviors [1], which may be probed in experiments and, hopefully, become useful in the engineering of quantum devices.

In condensed matter systems, inhomogeneities may lead to enhanced responses to external perturbations and/or to the emergence of local orders [2]. Remarkable examples include the large transport anisotropy observed at low-temperature in quantum Hall samples after the onset of electronic nematic phases [3], the colossal magneto-resistance in manganites [4], the appearance of striped phases in systems with competing interactions [5] as well as of pseudogap phases in high $T_{c}$ superconductors [6]. In all the above mentioned examples the relevant optimal inhomogeneities are dynamically generated [7]; furthermore, stripe and pseudogap phases are associated to the onset of local orders [5, 8].

To get control on the onset of complexity of a condensed matter system is desirable not only for understanding new emergent functionalities useful for the engineering of new materials and devices but also for discovering levels of theoretical description enabling to well separate the properties of global phases from the ones arising from phase competition; global average behaviors are, in fact, not helpful for this task [9]. Quantum devices provide a controllable setting (one can fabricate them!) to investigate the effects induced by inhomogeneities on the emergence of complex behaviors.

Quantum devices with built-in inhomogeneities may be realized with today's available technologies using either superconducting Josephson junction networks (JJN) [10] or ultra-

cold atoms in optical lattices [11]. Due to their versatility and to the great reliability of the fabrication technologies developed for their construction, JJNs and ultracold atoms in optical lattices are by now the prototype of complex physical systems exhibiting a variety of interesting physical behaviors, adjustable by acting only on very few external parameters and, as we shall point out, also by the pertinent engineering of the network's shape; in addition, they provide controllable settings to investigate the properties of granular superconductors or high $T_{c}$ superconductors [12] paving a very promising avenue in the engineering 
of quantum states of potential interest for processing quantum information [13].

A Josephson junction may be realized using two superconducting grains separated by an insulating layer; by an appropriate engineering of the insulating support, one can fabricate an inhomogeneous network of Josephson junctions with a given shape. Inhomogeneous JJNs [14, 15] have been studied for a long time with the aim of establishing the effect of space connectivity on superconductivity [16]. Recently, the appealing perspective to realize devices for the manipulation of quantum information stimulated the analysis of inhomogeneous planar JJNs with non conventional connectivity [17], engineered to sustain a topologically ordered ground state [18]. Furthermore, transport measurements on superconducting wire networks evidenced - even in pure systems with non-dispersive eigenstates interesting anomalies of the network critical current induced by the interplay between the network's geometry and topology with externally applied magnetic fields [19]. Furthermore, the theoretical analysis of rhombi chains has evidenced the exciting possibility of being able to detect $4 e$ superconductivity through measurements of the supercurrent in presence of a pertinent external magnetic field [20]. Here we shall address the properties of inhomogeneous JJNs fabricated on lattices with non-random regular inhomogeneities engineered to yield enhanced zero-voltage Josephson critical currents as well as local order on domains selected by the network's topology. We shall investigate in detail the paradigmatic example of a comb-shaped JJN, whose properties have been analyzed in [21, 22]. To fix the ideas, in Fig. 1 is reported the design of the device; there, the circles locate the position of the superconducting grains while the crosses represent the junction joining them.

Control over the network connectivity may be achieved also with ultracold atoms on optical lattices [23]. A one-dimensional optical lattice is realized using two counter-propagating laser beams arranged to create a periodic potential where bosons may tunnel between wells with a rate adjustable by tuning the laser's power controlling the height of the inter-well barrier. In this realization a lattice site is a minimum of the periodic potential while the links between neighbor vertices are provided by the barrier between wells. By suitably arranging more laser beams one is able to engineer the shape of the optical networks [24] allowing, in principle, for an experimental testing of the proprieties of inhomogeneous lattices such as the ones analyzed in [25, 26]. To fabricate bosonic Josephson junctions (BJJ) and networks (BJJN) one should require also that all the atoms in a given well are described by the same macroscopic wave function: a BJJ is obtained, then, by loading - at a temperature below 


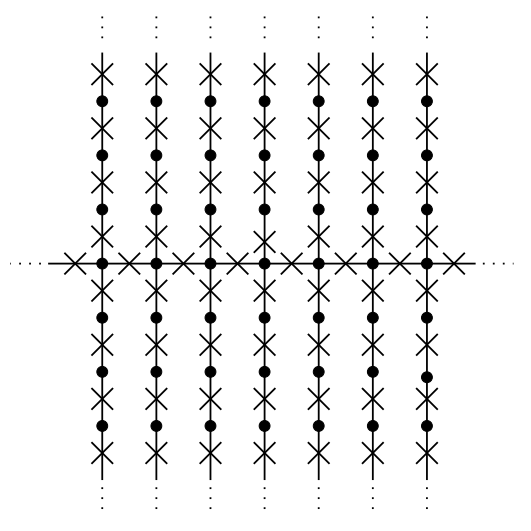

FIG. 1: The comb graph.

the Bose-Einstein condensation critical temperature - a condensate in each well of a double well potential [27] while the barrier separating the two condensates acts as a Josephson link [28]. With a multi-well optical potential, provided that the heights of the barriers are much higher than the condensate's chemical potential, one fabricates a BJJN (i.e., a lattice of weakly coupled condensates). In the following we shall evidence how bosons hopping on comb-shaped optical lattices undergo a spatial Bose-Einstein condensation on the comb's backbone; this happens even if the bosons are free and the network' s euclidean dimension is 1 .

The emergence of complex behaviors in the superconducting and bosonic arrays analyzed in this paper is determined solely by the pertinent engineering of the network's connectivity, since it is due only to the spectral properties of the adjacency matrix (i.e., of the matrix defined as $A_{i j}=1$ if $i$ and $j$ are vertices connected by a link, and $A_{i j}=0$ otherwise [29]), which fully characterizes the network's geometry and topology. In some very specific instances [30, 31], the spectrum of $A_{i j}$ contains a continuous set of states - the hidden spectrum - localized around domains selected by the network's topology with eigenvalues ranging continuously from a certain value $E_{0}$ up to the threshold of the continuum delocalized states $E_{d}$ : the spectrum is then effectively gapless even if, in the thermodynamic limit, there is a lowest eigenvalue $E_{0}$, confined away from $E_{d}$. In the following we shall evidence that it is this spectral anomaly - induced from the pertinent choice of the network's connectivity- which is responsible for the emergence of enhanced responses and local orderings in superconducting and bosonic networks, respectively. 
An hidden spectrum of the adjacency matrix emerges, for instance, when one analyzes bundled graphs [29] (i.e., those obtained by grafting a fiber graph to every point of a base graph) while, for graphs with constant coordination number (such as the Sierpinski gasket and the ladder graph), the adjacency matrix does not support any hidden spectrum [31]. In the following, we shall analyze the simple paradigmatic case of comb networks showing how the hidden spectrum of the adjacency matrix leads to unusual quantum behaviors such as the emergence of the spatial BEC on the comb's backbone for a Bose gas living on a combshaped optical lattice [30, 32] and of the enhanced responses observed for classical combshaped JJNs made of Niobium grains [21, 22][see Fig.1]. To better clarify our arguments, we find instructive to compare our results with those obtainable if the same devices were defined on a chain, since the latter is, after all, the simplest graph of euclidean dimension 1 .

In section 2 we determine the equations governing the properties of inhomogeneous superconducting and bosonic networks. Section 3 is devoted to the analysis of the spectrum of the adjacency matrix of a comb-shaped network and to a comparison of its spectrum with the one pertinent to a linear chain: there we see that, while the adjacency matrices of both graphs admit a continuum set of delocalized states starting at the same eigenvalue, the adjacency matrix of a comb supports an hidden spectrum. In section 4 we evidence how the states belonging to the hidden spectrum are responsible for the enhancement of the zerovoltage Josephson critical currents in superconducting JJNs as well as for the emergence of the spatial BEC in bosonic networks on domains pertinently selected by the network's topology. Section 5 is devoted to our conclusions and final remarks while the appendices report on some pertinent but rather lengthy computational details.

\section{MANY-BODY THEORY OF SUPERCONDUCTING AND BOSONIC SYS- TEMS ON GENERIC NETWORKS}

In this section we shall summarize the many-body description of superconducting and bosonic networks. Our purpose here is mainly to clarify the assumptions underlying the equations used in section 4 to characterize the complex behaviors emerging from a pertinent choice of the connectivity in JJNs and bosonic networks. Our approach uses the self- consistent Bogoliubov-De Gennes (BDG) equations [33] since they provide an unified framework to account for the description of fermionic and bosonic systems enabling to appreciate in a 
rather simple context the differences (and similarities) between these systems.

We shall describe first the microscopic theory of inhomogeneous superconducting networks realized by putting on each site of a graph a superconducting grain. On each island, the effects of the electron-phonon and Coulomb interactions are embodied in the BCS parameter and, furthermore, there exist a critical temperature $T_{B C S}$ such that for $T$ lesser than $T_{B C S}$ each grain becomes superconducting; of course, the entire network becomes superconducting at a lower temperature $T_{c}$ [34]. We, then, analyze ultracold atoms (spinless, for simplicity) in deep optical lattices and derive the set of equations describing both the excitations and the condensate's dynamics; in dealing with atomic systems one should only account for repulsive interactions between bosons as it is well known that attractive interactions lead to instabilities even in presence of confining traps [23].

\section{A. Superconducting networks}

Inhomogeneous fermionic systems with attractive interactions may be conveniently described by the BdG equations [33]: with a two-body point-like interaction $V\left(\vec{r}-\vec{r}^{\prime}\right)=$ $-\mathcal{V} \delta\left(\vec{r}-\vec{r}^{\prime}\right)$, the Hamiltonian is $\mathcal{H}=\mathcal{H}_{0}+\mathcal{H}_{1}$, where

$$
\mathcal{H}_{0}=\int d \vec{r} \sum_{\sigma} \Psi^{\dagger}(\vec{r} \sigma) H_{0} \Psi(\vec{r} \sigma)
$$

and

$$
\mathcal{H}_{1}=-\frac{\mathcal{V}}{2} \int d \vec{r} \sum_{\sigma \sigma^{\prime}} \Psi^{\dagger}(\vec{r} \sigma) \Psi^{\dagger}\left(\vec{r} \sigma^{\prime}\right) \Psi\left(\vec{r} \sigma^{\prime}\right) \Psi(\vec{r} \sigma) .
$$

In Eqs. (11) and (2), the $\Psi$ 's are fermionic operators, $\sigma= \pm$ is a spin index, and $H_{0}=$ $-\hbar^{2} \nabla^{2} / 2 m+U_{0}(\vec{r})-\mu$, where $\mu$ is the chemical potential and $U_{0}(\vec{r})$ is an external potential (we assume, as usual, that $U_{0}(\vec{r})$ is spin-independent and that no magnetic field is applied). When the fermions are not interacting, then $\mu=E_{F}$, where $E_{F}$ is the Fermi energy. In a self-consistent approach, one defines the effective Hamiltonian

$$
\begin{aligned}
\mathcal{H}_{e f f} & =\int d \vec{r} \sum_{\sigma}\left\{\Psi^{\dagger}(\vec{r} \sigma) H_{0} \Psi(\vec{r} \sigma)+\sum_{\sigma} U(\vec{r}) \Psi^{\dagger}(\vec{r} \sigma) \Psi(\vec{r} \sigma)\right\} \\
& +\int d \vec{r}\left\{\Delta(\vec{r}) \Psi^{\dagger}(\vec{r}+) \Psi^{\dagger}(\vec{r}-)+\Delta^{*}(\vec{r}) \Psi(\vec{r}-) \Psi(\vec{r}+)\right\}
\end{aligned}
$$

and, then, requires that the pair potential $\Delta(\vec{r}) \equiv \mathcal{V}\langle\Psi(\vec{r}+) \Psi(\vec{r}-)\rangle$ and the Hartree-Fock potential $U(\vec{r}) \equiv-\mathcal{V}\left\langle\Psi^{\dagger}(\vec{r} \sigma) \Psi(\vec{r} \sigma)\right\rangle$ are self-consistently determined from the solutions of 
the BdG equations

$$
\begin{gathered}
\epsilon_{\alpha} u_{\alpha}(\vec{r})=\left[H_{0}+U(\vec{r})\right] u_{\alpha}(\vec{r})+\Delta(\vec{r}) v_{\alpha}(\vec{r}) \\
\epsilon_{\alpha} v_{\alpha}(\vec{r})=-\left[H_{0}+U(\vec{r})\right] v_{\alpha}(\vec{r})+\Delta^{*}(\vec{r}) u_{\alpha}(\vec{r}),
\end{gathered}
$$

with $u_{\alpha}, v_{\alpha}$ satisfying

$$
\int d \vec{r}\left[\left|u_{\alpha}(\vec{r})\right|^{2}+\left|v_{\alpha}(\vec{r})\right|^{2}\right]=1
$$

One has then

$$
\Delta(\vec{r})=\mathcal{V} \sum_{\alpha} u_{\alpha}(\vec{r}) v_{\alpha}^{*}(\vec{r}) \tanh \left(\frac{\beta}{2} \epsilon_{\alpha}\right)
$$

and

$$
U(\vec{r})=-\mathcal{V} \sum_{\alpha}\left[\left|u_{\alpha}(\vec{r})\right|^{2} f_{\alpha}+\left|v_{\alpha}(\vec{r})\right|^{2}\left(1-f_{\alpha}\right)\right]
$$

where $f_{\alpha}=\left(e^{\beta \epsilon_{\alpha}}+1\right)^{-1}, \beta=1 / k_{B} T$ and the sums are only taken on positive $\in_{\alpha}$ 's. The chemical potential $\mu$ - when different from the Fermi energy - is determined from the normalization condition. Of course, when there is an external potential breaking the translational invariance, the pair potential is dependent on the position.

For spatially homogeneous superconducting networks the quantum number $\alpha$ is just the momentum $\vec{k}$ and, then, $\Delta=\Delta(\vec{r})$ and $U=U(\vec{r})$ do not depend on the position implying that $\epsilon_{\vec{k}}=\sqrt{\Delta^{2}+E_{\vec{k}}^{2}}$, where $E_{\vec{k}}=\hbar^{2} k^{2} / 2 m-\mu+U$. Furthermore, since $u_{\vec{k}}(\vec{r})=L^{-3 / 2} U_{\vec{k}} e^{i \vec{k} \cdot \vec{r}}$ and $v_{\vec{k}}(\vec{r})=L^{-3 / 2} V_{\vec{k}} e^{i \vec{k} \cdot \vec{r}}$ with $L^{3}$ being the volume, one gets $U_{\vec{k}}^{2}=(1 / 2)\left[1+E_{\vec{k}} / \epsilon_{\vec{k}}\right]$ and $V_{\vec{k}}^{2}=(1 / 2)\left[1-E_{\vec{k}} / \in_{\vec{k}}\right]$. With the BCS interaction (i.e., $\mathcal{V}=V_{B C S}$ if $\left|\hbar^{2} k^{2} / 2 m-E_{F}\right|$, $\left|\hbar^{2} k^{2} / 2 m-E_{F}\right|<\hbar \omega_{D}$ and $\mathcal{V}=0$ otherwise, $\omega_{D}$ being the Debye frequency) and $U=0$ (which is a good solution of the self-consistent equation for $U$ if $n(0) V_{B C S} \ll 1$ ), one has $\mu=E_{F}$ together with the celebrated BCS gap-equation

$$
1=\frac{n(0) V_{B C S}}{2} \int_{-\hbar \omega_{D}}^{\hbar \omega_{D}} \frac{d E}{\sqrt{\Delta^{2}+E^{2}}} \tanh \left(\frac{\beta}{2} \sqrt{\Delta^{2}+E^{2}}\right)
$$

$n(0)$ is the density of states per volume and spin direction at the Fermi energy. For inhomogeneous networks, instead, one should regard the external potential $U_{0}(\vec{r})$ as representing the insulating barriers between the grains and take self-consistently into account its effects.

To obtain a discrete version of the BdG equations ( $\mathrm{LBdG}$ ) suitable to describe the classical superconducting JJNs fabricated in [21], one makes the ansatz that the eigenfunctions of the continuous BdG equations [33] may be written in a tight binding form as $u_{\alpha}(\vec{r})=$ $\sum_{i} u_{\alpha}(i) \phi_{i}(\vec{r})$ and $v_{\alpha}(\vec{r})=\sum_{i} v_{\alpha}(i) \phi_{i}(\vec{r}) ; i$ labels the position of a superconducting island 
while the contribution of the electronic states participating to superconductivity in a given island is effectively described by a field $\phi_{i}(\vec{r})$, whose specific form depends only on the geometry of the islands and on the fabrication parameters of the connecting junctions. The assumption that $\phi_{i}(\vec{r})$ does not depend on $\alpha$ amounts to account only for contributions coming from electrons near the Fermi surface. The LBdG equations then read

$$
\begin{gathered}
\epsilon_{\alpha} u_{\alpha}(i)=\sum_{j} \epsilon_{i j} u_{\alpha}(j)+\Delta(i) v_{\alpha}(i) \\
\epsilon_{\alpha} v_{\alpha}(i)=-\sum_{j} \epsilon_{i j} v_{\alpha}(j)+\Delta^{*}(i) u_{\alpha}(i) .
\end{gathered}
$$

where $u_{\alpha}$ and $v_{\alpha}$ satisfy to

$$
\sum_{i}\left[\left|u_{\alpha}(i)\right|^{2}+\left|v_{\alpha}(i)\right|^{2}\right]=1
$$

The matrix $\epsilon_{i j}$ is defined by

$$
\epsilon_{i j}=-t A_{i j}+U(i) \delta_{i j}-\tilde{\mu} \delta_{i j}
$$

with $A_{i j}$ being the adjacency matrix of the network,

$$
\tilde{\mu}=\mu-\int d \vec{r} \phi_{i}(\vec{r})\left(-\hbar^{2} \nabla^{2} / 2 m\right) \phi_{i}(\vec{r})
$$

and

$$
t=-\int d \vec{r} \phi_{i}(\vec{r})\left[-\hbar^{2} \nabla^{2} / 2 m+U_{0}(\vec{r})\right] \phi_{j}(\vec{r}) .
$$

Self-consistency requires

$$
\Delta(i)=\tilde{\mathcal{V}} \sum_{\alpha} u_{\alpha}(i) v_{\alpha}^{*}(i) \tanh \left(\frac{\beta}{2} \epsilon_{\alpha}\right)
$$

and

$$
U(i)=-\tilde{\mathcal{V}} \sum_{\alpha}\left[\left|u_{\alpha}(i)\right|^{2} f_{\alpha}+\left|v_{\alpha}(i)\right|^{2}\left(1-f_{\alpha}\right)\right],
$$

where $\tilde{\mathcal{V}} \equiv \mathcal{V} \phi^{2}\left(\vec{r}=\vec{r}_{i}\right)$ is assumed to be independent of $i$. The network's topology and geometry is encoded in the term $-t A_{i j}$ appearing in the definition of the matrix $\epsilon_{i j}$ given in Eq. (13), while the specific values of $t$ and $\tilde{\mathcal{V}}$ depend - as a result of our ansatz on the form of the eigenfunctions of the BdG equations- only on the $\phi_{i}(\vec{r})$.

To justify the assumptions involved in the derivation of Eqs. (10) and (11), one should observe that, for the JJN device described in [21], capacitive (inter islands and with a ground) 
effects are negligible, that the total number of electrons on the island $\mathcal{N}$ is much larger than the number of electrons tunneling through the Josephson junction and that all the islands contain approximately the same $\mathcal{N}(\mathcal{N}(i) \equiv \mathcal{N})$. Furthermore, the islands are big enough to support the same superconducting gap of the bulk material; as a result, one may require $\phi_{i}(\vec{r})$ to be position-independent on each island except for a small region near the junction and to be the same on each island with a normalization given by $\int d \vec{r} \phi_{i}^{2}(\vec{r})=\mathcal{N}(i) \equiv \mathcal{N}$ and $\int d \vec{r} \phi_{i}(\vec{r}) \phi_{j}(\vec{r}) \approx 0$ for $i \neq j$; in our derivations we set $\mathcal{N} \equiv 1$. As a result $t \approx E_{J}=(\hbar / 2 e) I_{c}$, where $E_{J}$ is the nominal value of the Josephson energy of all the junctions in the network while $I_{c}$ is the bare zero-voltage Josephson critical current of each junction. In section IV we shall provide an explicit solution of the BdG equations (10) and (11) describing superconducting JJNs fabricated on a comb-shaped insulating support [21], while in App. A a solution of the LBdG equations for a Josephson junction chain is provided.

\section{B. Bosonic networks}

The full quantum Hamiltonian pertaining to a (spinless) bosonic gas in an optical potential is $\mathcal{H}=\mathcal{H}_{0}+\mathcal{H}_{1}$, where

$$
\mathcal{H}_{0}=\int d \vec{r} \Phi^{\dagger}(\vec{r}) H_{0} \Phi(\vec{r})
$$

and

$$
\mathcal{H}_{1}=\frac{g_{0}}{2} \int d \vec{r} \Phi^{\dagger}(\vec{r}) \Phi^{\dagger}(\vec{r}) \Phi(\vec{r}) \Phi(\vec{r})
$$

In Eqs. (18) and (19),$\Phi$ is bosonic operator, and $H_{0}=-\hbar^{2} \nabla^{2} / 2 m+U_{0}(\vec{r})-\mu ; U_{0}(\vec{r})$ is the external potential, which is usually the sum of the optical lattice and of the magnetic trap potential; for simplicity, we do not consider terms accounting for the effects of harmonic traps. In writing Eqs. (18) and (19), the standard $s$-wave scattering approximation has been used: i.e., the two-body potential is written as $V\left(\vec{r}-\overrightarrow{r^{\prime}}\right)=g_{0} \delta\left(\vec{r}-\overrightarrow{r^{\prime}}\right)$, where $g_{0}=$ $4 \pi \hbar^{2} a / m>0$, with $a$ being the $s$-wave scattering length and $m$ the atomic mass. The dynamics of the bosonic field is described by the well known equation [23]

$$
i \hbar \frac{\partial}{\partial t} \Phi=\left[H_{0}+g_{0} \Phi^{\dagger} \Phi\right] \Phi
$$

from which the Gross-Pitaevskii equation for the condensate wavefunction $\psi=\langle\Phi\rangle$ is usually derived [23]. 
The bosonic counterpart of the BdG Eqs. (44) and (5) is provided by the Bogoliubov equations for the condensate's excitations [23]: to derive them, one usually writes $\Phi=\psi+\delta \Phi$ with

$$
\delta \Phi=\sum_{\alpha}\left[u_{\alpha}(\vec{r}) a_{\alpha} e^{-i \epsilon_{\alpha} t / \hbar}-v_{\alpha}^{*}(\vec{r}) a_{\alpha}^{\dagger} e^{i \epsilon_{\alpha} t / \hbar}\right]
$$

where $a_{\alpha}$ are operators destroying bosons in the excited state $\alpha$. Using Eq. (20) and keeping only terms linear in the fluctuation field $\delta \Phi$, one finds

$$
\begin{aligned}
& \epsilon_{\alpha} u_{\alpha}(\vec{r})=\left[H_{0}+2 g_{0} n_{0}(\vec{r})\right] u_{\alpha}(\vec{r})-g_{0} n_{0}(\vec{r}) v_{\alpha}(\vec{r}) \\
& \epsilon_{\alpha} u_{\alpha}(\vec{r})=\left[-H_{0}-2 g_{0} n_{0}(\vec{r})\right] u_{\alpha}(\vec{r})+g_{0} n_{0}(\vec{r}) v_{\alpha}(\vec{r})
\end{aligned}
$$

where $n_{0}(\vec{r})=|\psi(\vec{r})|^{2}$ is the condensate density [23]. Notice that $u_{\alpha}$ and $v_{\beta}$ satisfy now to the condition

$$
\int d \vec{r}\left[\left|u_{\alpha}(\vec{r})\right|^{2}-\left|v_{\alpha}(\vec{r})\right|^{2}\right]=1 .
$$

In the analysis of a bosonic system one has to use, in addition to the BDG equations, the Gross-Pitaevskii equation for the condensate. To do this, one should observe that, when the power laser is high enough, the bosonic field $\Phi$ may be approximated by means of the tight-binding approximation [35] as

$$
\Phi(\vec{r}, t)=\sum_{j} b_{j}(t) \phi_{j}(\vec{r})
$$

substituting this ansatz in the full Hamiltonian, one gets (in the non interacting limit) a simple tight-binding model described by

$$
H=-t \sum_{i j} A_{i j} b_{i}^{\dagger} b_{j}
$$

where the coefficient $t$ is given by Eq. (15). In Eq. (26) $, i, j$ denote the minima of the optical lattice (i.e., sites of the network) and $b_{j}^{\dagger}\left(b_{j}\right)$ is the bosonic operator which creates (destroys) a boson at site $j$. The filling, i.e., the average number of particles per site, is defined as $f=N_{T} / N_{S}$, where $N_{T}$ is the total number of bosons and $N_{S}$ is the total number of sites.

Eq. (26) is the pertinent equation to investigate in order to ascertain if, for a gas of ultracold bosons, BEC emerges as a result of the network's inhomogeneity. When BEC occurs in each well, each pair of neighbouring wells acts as a bosonic junction with Josephson energy given by

$$
E_{J} \approx 2 t f
$$


In the next sections we shall show that a condensate indeed emerges when bosons hop on comb-shaped networks.

\section{SPECTRUM OF A QUANTUM PARTICLE HOPPING ON COMB-SHAPED NETWORKS}

In section 2 we evidenced how the equations describing superconducting and bosonic networks depend on the adjacency matrix characterizing the network's connectivity. In this section we shall review [30] the main results concerning the spectrum of the adjacency matrix describing the connectivity of a comb network.

A comb (see Fig. 1) is made of one-dimensional chains (fingers) grafted periodically on a linear chain (backbone). Each site of the comb can be naturally labeled by introducing two integer indices $(x, y)$, where $x=0, \cdots, L_{1}$ labels the different fingers and $y=0, \cdots, L_{2}$ provides the distance from the backbone. Each site on the finger is linked to two neighbors whereas each site of the backbone has four neighbors.

The topology of the network is fully described by the adjacency matrix $A_{x, y ; x^{\prime}, y^{\prime}}$ which equals 1 if $\left(x, y ; x^{\prime}, y^{\prime}\right)$ is a link and 0 otherwise. A quantum particle hopping on a comb is, then, described by the Hamiltonian

$$
H=-t \sum_{x, y ; x^{\prime}, y^{\prime}} A_{x, y ; x^{\prime}, y^{\prime}} b_{x, y}^{\dagger} b_{x^{\prime}, y^{\prime}}
$$

The single-particle energy spectrum is found by solving the eigenvalue equation [30, 31, 32]:

$$
-t \sum_{x^{\prime}, y^{\prime}} A_{x, y ; x^{\prime}, y^{\prime}} \psi_{E}\left(x^{\prime}, y^{\prime}\right)=E \psi_{E}(x, y)
$$

on a comb $A_{x, y ; x^{\prime}, y^{\prime}}$ is given by

$$
A_{x, y ; x^{\prime}, y^{\prime}}=\left(\delta_{x, x^{\prime}+1}+\delta_{x, x^{\prime}-1}\right) \delta_{y, 0} \delta_{0, y^{\prime}}+\left(\delta_{y, y^{\prime}+1}+\delta_{y, y^{\prime}-1}\right) \delta_{x, x^{\prime}}
$$

In the following, we shall determine the spectrum of a quantum particle on the finite $L_{1} \times L_{2}$ comb and, only at the end, take the limit $L_{1}, L_{2} \rightarrow \infty$. On a finite $L_{1} \times L_{2}$ comb - using the adjacency matrix (30) - the eigenvalue equation (29) reads:

$$
-t \sum_{x^{\prime}=0}^{L_{1}-1} \sum_{y^{\prime}=0}^{L_{2}-1}\left[\left(\delta_{x, x^{\prime}+1}+\delta_{x, x^{\prime}-1}\right) \delta_{y, 0} \delta_{0, y^{\prime}}+\left(\delta_{y, y^{\prime}+1}+\delta_{y, y^{\prime}-1}\right) \delta_{x, x^{\prime}}\right] \psi\left(x^{\prime}, y^{\prime}\right)=E \psi(x, y)
$$


Without loss of generality one may take $x$ and $y$ to be positive integers, since, due to periodic boundary conditions, $(0,0) \equiv\left(L_{1}, 0\right)$ and $(x, 0) \equiv\left(x, L_{2}\right)$. The total number of sites is then $N_{S}=L_{1} \times L_{2}$.

By exploiting the translation invariance along the backbone, a Fourier transform in the variable $x$ reduces Eq. (31) to a one-dimensional eigenvalue problem for a quantum particle hopping on the comb's fingers. In fact, upon defining

$$
\psi(k, y)=\sum_{x} e^{i k x} \psi(x, y)
$$

with $k=2 \pi n / L_{1}, n=0, \ldots L_{1}-1$, the eigenvalue equation (31) becomes:

$$
-t \sum_{k^{\prime}=0}^{L_{1}-1} \sum_{y^{\prime}=0}^{L_{2}-1}\left[2 \cos (k) \delta_{y, 0} \delta_{0, y^{\prime}} \delta_{k, k^{\prime}}+\left(\delta_{y, y^{\prime}+1}+\delta_{y, y^{\prime}-1}\right) \delta_{k, k^{\prime}}\right] \psi\left(k^{\prime}, y^{\prime}\right)=E \psi(k, y)
$$

Since Eq. (33) is diagonal in $k$, it may be written as:

$$
-t \sum_{y^{\prime}}\left[2 \cos \left(k_{0}\right) \delta_{y, 0} \delta_{0, y^{\prime}}+\left(\delta_{y, y^{\prime}+1}+\delta_{y, y^{\prime}-1}\right)\right] \psi\left(y^{\prime}\right)=E \psi(y)
$$

where $\psi(k, y)=\delta\left(k-k_{0}\right) \psi(y)$ with $k_{0}=2 \pi n / L_{1}, n=0, \ldots, L_{1}-1$. Equation (34) describes then a one-dimensional quantum particle interacting with a potential located on the backbone, $V\left(k_{0}\right)=-2 t \cos \left(k_{0}\right)$.

To determine the eigenvalues and eigenvectors of Eq. (34) one may look for solutions of the form

$$
\begin{aligned}
\psi(y)=A_{0} \cos (h y+\alpha) & \text { for } \quad-2 t \leq E=-2 t \cos (h) \leq 2 t ; \\
\psi(y)=A_{-} e^{-h y}+B_{-} e^{h y} & \text { for } \quad E=-t\left(e^{h}+e^{-h}\right)<-2 t \\
\psi(y)=A_{+}(-1)^{y} e^{-h y}+B_{+}(-1)^{y} e^{h y} & \text { for } \quad E=t\left(e^{h}+e^{-h}\right)>2 t
\end{aligned}
$$

To fix both the free parameters and the eigenvalues $E$ one requires $\psi(y)$ to be normalizable and to be a solution of the eigenvalue equation in $y=0$ and $y=L_{2}-1$. These points are the only ones where Eq. (34) is not identically satisfied, yielding two equations to determine the two free parameters. Since for a given value of $k_{0}$ there are $L_{2}$ different eigenvalues, the spectrum will consist of $L_{1} \cdot L_{2}$ states and it can be divided in three regions: $\sigma_{0}$ and $\sigma_{ \pm}$[30].

- $\sigma_{0}$ 
$\sigma_{0}$ is the part of the spectrum corresponding to delocalized states with energies between $-2 t$ and $2 t$. Requiring the wavefunction (35) to be a solution of the eigenvalue equation (34) in $y=L_{2}-1$ and $y=0$ yields

$$
\begin{aligned}
& \cos \left[h\left(L_{2}-2\right)+\alpha\right]+\cos (\alpha)=2 \cos (h) \cos \left[h\left(L_{2}-1\right)+\alpha\right] \\
& \cos \left[h\left(L_{2}-1\right)+\alpha\right]+\cos (h+\alpha)+2 \cos \left(k_{0}\right) \cos (\alpha)=2 \cos (h) \cos (\alpha),
\end{aligned}
$$

implying that there are odd eigenfunctions with $\alpha=\pi / 2, h=2 \pi m / L_{2}$ and $m=$ $1, \ldots, L_{2} / 2-1$ and $L_{2} / 2$ even solutions, obtained by

$$
-\cos \left(k_{0}\right) \cot \left(h L_{2} / 2\right)=\sin (h)
$$

Equation (39) can be solved graphically. In the large $L_{2}$ limit the allowed values for $h$ are: $h \approx \pi(2 m-1) / L_{2}$, with $m=1, \ldots, L_{2} / 2$. For each value of $k_{0}$ there are $L_{2}-1$ eigenvalues of type (35) with energy $E=-2 t \cos (h)$ and wavefunctions $\psi(x, y)=e^{i k_{0} x} \sin (h y)$ and $\psi(x, y)=e^{i k_{0} x} \cos (h|y|+\alpha)$, with $h$ and $\alpha$ satisfying Eq. (38). Thus, the fraction of states in this spectral region is $f=L_{1}\left(L_{2}-1\right) / L_{1} \cdot L_{2}$. Of course, $f$ tends to 1 in the limit $L_{1}$, $L_{2} \rightarrow \infty$ and the density of states is given by [31]:

$$
\rho_{0}(E) d E=d n=L_{1} d\left(L_{2} h / 2 \pi\right)=L_{1}\left(L_{2}-1\right) \frac{d E}{\pi \sqrt{4 t^{2}-E^{2}}},
$$

just as for a particle hopping on a linear chain.

- $\sigma_{-}$

$\sigma_{-}$is the part of the spectrum corresponding to localized states with energies $E<-2 t$. Requiring that the wavefunction (36) is a solution of the eigenvalue equation (34) in $y=$ $L_{2}-1$ and $y=0$ yields now

$$
\begin{aligned}
& A_{-} e^{-h\left(L_{2}-2\right)}+B_{-} e^{h\left(L_{2}-2\right)}+\left(A_{-}+B_{-}\right)=\left(e^{h}+e^{-h}\right)\left(A_{-} e^{-h\left(L_{2}-1\right)}+B_{-} e^{h\left(L_{2}-1\right)}\right) \\
& A_{-} e^{-h\left(L_{2}-1\right)}+B_{-} e^{h\left(L_{2}-1\right)}+\left(A_{-} e^{-h}+B_{-} e^{h}\right)+2 \cos \left(k_{0}\right)\left(A_{-}+B_{-}\right)=\left(e^{h}+e^{-h}\right)\left(A_{-}+B_{-}\right),
\end{aligned}
$$

leading to:

$$
\cos \left(k_{0}\right) \operatorname{coth}\left(h L_{2} / 2\right)=\sinh (h)
$$

Equation (42) can be solved graphically, yielding a real solution only if $\cos \left(k_{0}\right)>0$. The density of states is then given by

$$
\rho_{-}(E) d E=d n=d\left(L_{1} k_{0} / 2 \pi\right)=L_{1} \frac{|E| d E}{2 \pi \sqrt{8 t^{2}-E^{2}} \sqrt{E^{2}-4 t^{2}}} .
$$


- $\sigma_{+}$

$\sigma_{+}$is the part of the spectrum corresponding to localized states with energies $E>2 t$. The parameters $A_{+}$and $B_{+}$are fixed by requiring again that the wavefunction (37) is a solution of the eigenvalue equation (34) in $y=L_{2}-1$ and $y=0$. This requirement yields a set of equations similar to those of Eq. (42), leading to $\cos \left(k_{0}\right) \operatorname{coth}\left(h L_{2} / 2\right)=-\sinh (h)$, which supports a real solution only if $\cos \left(k_{0}\right)<0$ while, for $\cos \left(k_{0}\right)=0$, one gets the constant solution $\psi(y)=1$ with energy $E=0$. The density of states in this spectral region is given by

$$
\rho_{+}(E) d E=d n=d\left(L_{1} k_{0} / 2 \pi\right)=L_{1} \frac{|E| d E}{2 \pi \sqrt{8 t^{2}-E^{2}} \sqrt{E^{2}-4 t^{2}}} .
$$

The union of $\sigma_{-}$(i.e., states with $\left.E<-2 t\right)$ and $\sigma_{+}(E>2 t)$ forms the hidden spectrum and is the part of the spectrum corresponding to localized states; each spectral region contains $L_{1} / 2$ states and, thus, $L_{1}$ states belong to the hidden spectrum. Taking the limit $L_{2}$ and $L_{1} \rightarrow \infty$, from Eq. (42), one easily verifies that the states of the hidden spectrum satisfy $\sinh (h)=\cos \left(k_{0}\right)$ and correspond to energy eigenvalues $E=-2 t \sqrt{1+\cos ^{2}\left(k_{0}\right)}$ for $\sigma_{-}$and $E=2 t \sqrt{1+\cos ^{2}\left(k_{0}+\pi / 2\right)}$ for $\sigma_{+}$. As the comb's size gets bigger, almost all the states- i.e., all the states apart from a set of measure zero - belong to $\sigma_{0}$; in fact, since $\int_{\sigma_{0}} \rho_{0}(E) d E=L_{1} \cdot\left(L_{2}-1\right)$, the normalized density of states belonging to $\sigma_{0}$ tends to 1 while, for what concerns the hidden spectrum, one has $\lim _{L_{1}, L_{2} \rightarrow \infty} \int_{\sigma_{ \pm}} \rho_{ \pm}(E) d E=0$. Since the hidden spectrum does not contribute to the normalized density of states $\rho(E)$ of the pure hopping model on the comb, one has:

$$
\rho(E)=\frac{1}{\pi \sqrt{4 t^{2}-E^{2}}} .
$$

Normalizing the density of states of the lower hidden spectrum to $L_{1}$, one obtains for $E \in \sigma_{-}$:

$$
\frac{1}{L_{1}} \rho_{-}(E)=\frac{|E|}{2 \pi \sqrt{8 t^{2}-E^{2}} \sqrt{E^{2}-4 t^{2}}}
$$

An analogous equation holds for the spectral region $E \in \sigma_{+}$. The density of states can then be plotted as in Fig. 2, where the pertinent normalizations for the continuous and hidden part of the spectrum have been used.

The lowest energy eigenvalue in the normalized density of states is $E_{d}=-2 t$; but, this is not the lowest energy attainable by a particle hopping on a comb since there is a lowest localized eigenstate belonging to $\sigma_{-}$whose eigenvakue is given by:

$$
E_{0}=-2 \sqrt{2} t
$$




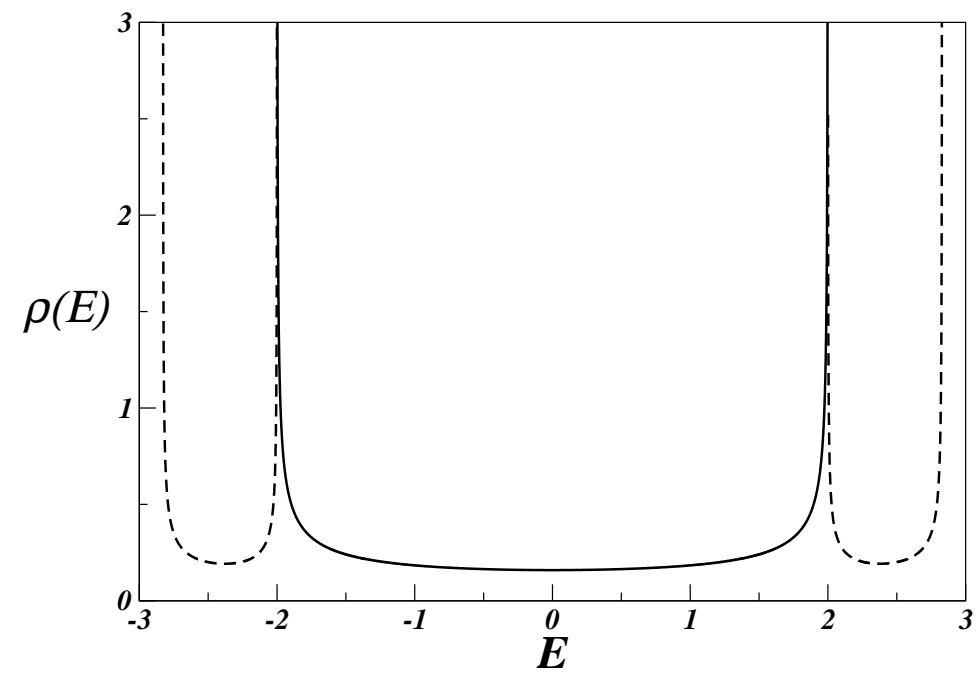

FIG. 2: The density of states of the Hamiltonian (28) in units of $t$. The solid line indicates the continuous part of the spectrum $\rho_{0}$, normalized to $L_{1} \cdot\left(L_{2}-1\right)$. The dashed lines denote $\rho_{ \pm}$, normalized to $L_{1}$.

In fact, for $L_{2} \rightarrow \infty$, since the energy is a decreasing function of $h$, the lowest energy level of $\sigma_{-}$is attained when $\cos \left(k_{0}\right)=1$.

Of course, $E_{0}<E_{d}$ and this should indicate that the spectrum is gapped. However, one does not find an energy gap between $E_{0}$ and $E_{d}$, since, for each value of $k_{0}\left(\cos \left(k_{0}\right)>0\right)$ there is a solution of Eq. (42) with a different energy in the interval $\left[E_{0}, E_{d}\right]$. In a finite comb of $L_{1} \times L_{2}$ sites there are $L_{1} / 2$ solution of this type and, as $L_{1} \rightarrow \infty$, these solutions fill densely the interval $\left[E_{0}, E_{d}\right]$. These spectral proprieties should be contrasted with the ones arising when non interacting quantum particles are trapped in a harmonic well.

From Eqs. (36) and (41) it is also possible to show that, when $L_{1}, L_{2} \rightarrow \infty$, the eigenvector corresponding to the lowest energy eigenvalue is

$$
\psi_{E_{0}}(x, y)=\frac{1}{2^{1 / 4}} e^{-|y| / \xi}
$$

where $\xi=1 / h=1 / \log (1+\sqrt{2})$ is the parameter accounting for the localization around the backbone. In Fig. 3 we plot the ground-state wavefunction as a function of the distance from the backbone; the plot well evidences the exponential localization only around the comb's backbone.

Although not explicitly imposed, the condition of the continuity of the discrete gradient 


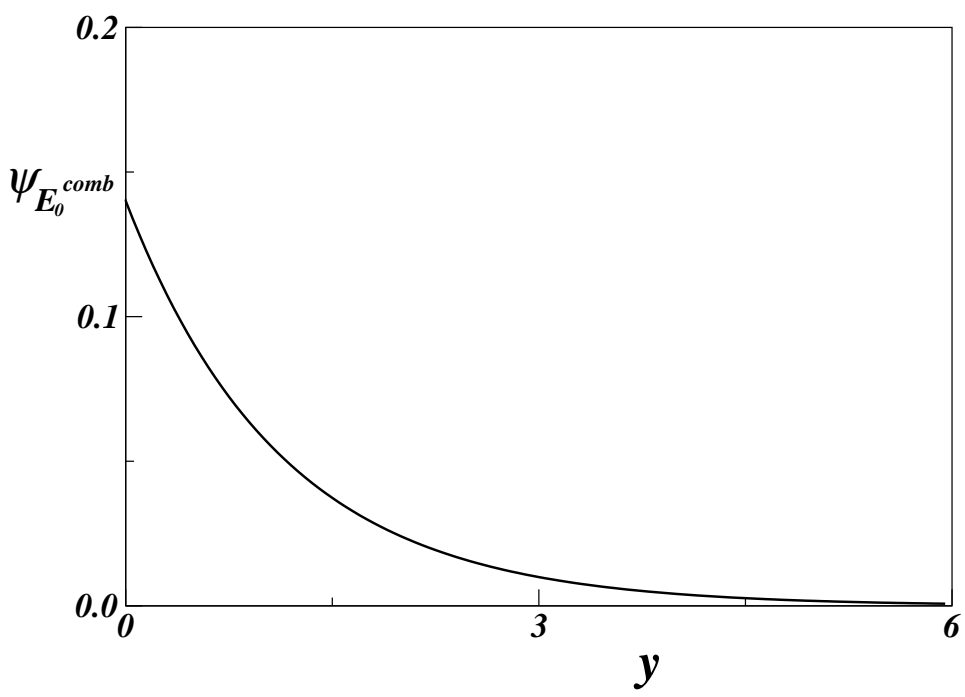

FIG. 3: The normalized ground-state wavefunction of the comb network as a function of the distance $y$ from the origin for a comb with $51 \times 51$ sites.

holds at the points $(0, y)$. Of course one cannot ask for the continuity of the linear derivatives along $x$ or $y$ since it is just this discontinuity which allows a particle moving on the finger to hop in the direction of the backbone.

A similar analysis may be carried to determine the spectrum of a quantum particle hopping on different comb-like networks, such as the star-comb, mini-comb, and semi-comb depicted in Fig. 4. It is not difficult to convince one-self that, for a pertinent choice of boundary conditions, the adjacency matrix describing the connectivity of these bundled graphs admits also an hidden spectrum.

In the following we shall report only the lowest energy eigenvalues pertaining to quantum particles hopping on these networks. For a star-comb [36] one has

$$
E_{0}^{s c}=-t \frac{(p-2)+p \sqrt{p}}{p-1},
$$

where $p$ is the number of arms on each star. For the semi-comb, one finds

$$
E_{0}^{s e m i}=-2 \sqrt{\phi} t
$$

where $\phi=(1+\sqrt{5}) / 2 \simeq 1.618$ is the golden section. For a mini-comb, one finds

$$
E_{0}^{m i n i}=-(1+\sqrt{1+p}) t
$$



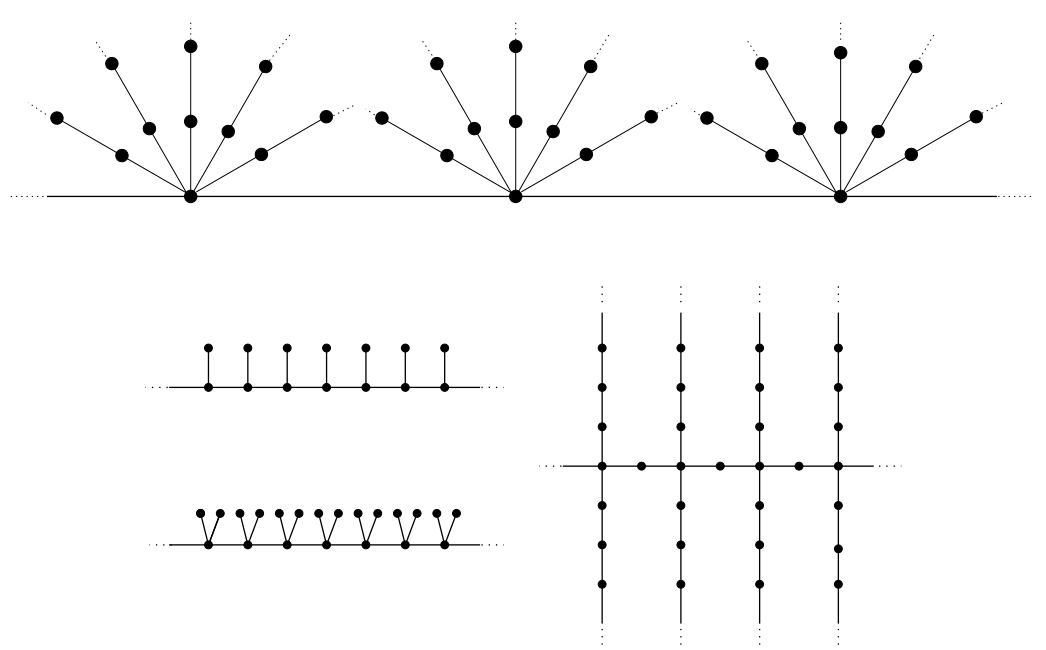

FIG. 4: Top: The star-comb network. Bottom Left: Two "mini"-comb networks (top: $p=1$; bottom: $p=2$ ). Bottom Right: the "semi"-comb network.

As we shall see in the following, the value of the ground-state energy is intimately tied to the critical temperature at which quantum bosons hopping on comb-networks undergo a spatial Bose-Einstein condensation.

\section{COMPLEX BEHAVIORS EMERGING FROM THE NETWORK'S CONNEC- TIVITY}

In this section we review how the pertinent choice of the network's connectivity leads to the emergence of new phenomena in quantum devices realized with superconducting JNNs [22] and cold atoms in optical lattices [30, 32]. Our subsequent analysis well evidences that new emerging phenomena are possible only if the network's connectivity is described by an adjacency matrix supporting an hidden spectrum. As we shall see, the states belonging to the hidden spectrum induce in a discrete many body system the onset of a new relevant energy scale and enhance the number of states which can be occupied by quantum particles at low energy. 


\section{A. Enhanced Josephson critical currents in a comb-shaped JJN}

In the following, we use the LBdG equations derived in Section 2 to compute the zerovoltage Josephson critical currents of junctions located on Josephson linear chains and comb-shaped Josephson networks. Using the eigenfunctions of the LBdG equations, a selfconsistent computation yields for both systems the gap function, the chemical potential and the quasi particle spectrum. Our analysis evidences that, on the backbone of a combshaped JJN, the BCS equations are satisfied with a renormalized value of the Josephson energy. Then, we compute the zero-voltage Josephson critical currents $I_{c}$ on the comb's backbone and compare our results for $I_{c}$ with the outcomes of the experimental measurements presented in [21, 22] and summarized in Fig.5.

For a comb network with $L \times L$ islands (see Fig.1), one finds a solution of the LBdG equations (10) and (11) where both the Hartree-Fock potential $U(i)$ and the gap function $\Delta(i)$ are position dependent. The eigenvalue equation

$$
-E_{J} \sum_{j} A_{i j} \psi_{\alpha}(j)=e_{\alpha} \psi_{\alpha}(i)
$$

leads to the emergence of the hidden spectrum analyzed in section 3.

For a crude analytical estimate, one may require that, away from the backbone, the fingers may be regarded as a linear chain with uniform potentials (i.e., $\Delta(i)=\Delta_{c}$ and $U(i)=U_{c}$ ). To get coupled equations for $\Delta_{b}, \Delta_{c}, U_{b}$, and $U_{c}$, one writes the LBdG equations (10) and (11) on a generic backbone's grain $i$. Upon setting $u_{\alpha}(i)=U_{\alpha} \psi_{\alpha}(i)$ and $v_{\alpha}(i)=V_{\alpha} \psi_{\alpha}(i)$, with $U_{\alpha}^{2}+V_{\alpha}^{2}=1$, the self-consistency equation for $U$ implies that, at $T=0, U_{b} \approx U_{c}-\frac{\tilde{\mathcal{V}} C_{0}^{2}}{2}$; upon requiring $\tilde{\mu} \approx U_{b}$ one immediately sees that, due to the localized modes of the hidden spectrum, the chemical potential on the comb's backbone is smaller than the one measured on the chain.

By substituting the wavefunctions of the eigenstates of the hidden spectrum [30] in Eqs. (10) and (11) and using $\tilde{\mu} \approx U_{b}$ one gets

$$
\Delta_{b}=\Delta_{c}+\frac{\Delta_{b} \tilde{\mathcal{V}}}{\pi} \cdot \int_{0}^{\pi / 2} d k \frac{\cos k}{\epsilon_{k} \sqrt{1+\cos ^{2} k}} \cdot \tanh \left(\frac{\beta}{2} \epsilon_{k}\right) .
$$

where $\epsilon_{k}=\sqrt{\Delta_{b}^{2}+4 E_{J}^{2}\left(1+\cos ^{2} k\right)}$. The hidden spectrum eigenstates contribute also to the gap function $\Delta_{b}$ through the second term in the rhs of Eq. (53): without an hidden spectrum, $\Delta_{b}$ equals $\Delta_{c}$. 
When $E_{J} \gg \Delta_{b}, \Delta_{c}$, Eq. (153), at $T=0$, yields

$$
\frac{\Delta_{b}(T=0)}{\Delta_{c}(T=0)}=\frac{1}{1-\frac{\eta_{c} \tilde{\mathcal{V}}}{2 \pi E_{J}}} \equiv \mathcal{K}
$$

where $\eta_{c} \equiv(1 / \sqrt{2}) \log (1+\sqrt{2})$. Furthermore, at low temperatures,

$$
\Delta_{b}(T) / \Delta_{c}(T) \approx \Delta_{b}(T=0) / \Delta_{c}(T=0)
$$

Using the parameters $E_{J}$ and $\tilde{\mathcal{V}}$ obtained from the measurements carried on the JJ chain (see Appendix $\mathrm{A})$, one gets $\mathcal{K} \approx 1.13$.

Upon requiring that the $T=0$ backbone's gap function has a BCS like functional formi.e., $\Delta_{b}(T=0)=8 \bar{E}_{J} e^{-2 \pi \bar{E}_{J} / \overline{\mathcal{V}}}$, with $\bar{E}_{J}$ and $\overline{\tilde{\mathcal{V}}}$ being the renormalized Josephson energy and the renormalized interaction term- one is able to estimate the renormalization of the Josephson coupling within the LBdG approach. Namely, one has

$$
\bar{E}_{J}=\mathcal{K} E_{J} ; \quad \overline{\tilde{\mathcal{V}}}=\mathcal{K} \tilde{\mathcal{V}}
$$

which embodies the effects of the hidden spectrum on the Josephson current. In Fig. 5 we plot, as a function of the normalized temperature, the values of $I_{c}$ measured in [21] (squares) and the values of $I_{c}$ obtained from the Ambegaokar-Baratoff [37] formula using both the renormalized coupling given by Eq. (56) and the gap function along the backbone for the comb-like JJN studied in [21, 22] (solid curve): the agreement of the results of the LBDG analysis with the outcomes of experiments is very good at low temperature.

\section{B. Spatial BEC of bosons hopping on a comb}

The thermodynamic properties of non-interacting bosons hopping on a comb evidences the emergence of a spatial BEC on the backbone even if the euclidean dimension of a comb is 1 [30, 31]. In this section we shall review the argument showing how this emergent behavior follows from the existence of an hidden part in the spectrum of the adjacency matrix describing the comb's connectivity; in fact, the presence of a dense set of states filling the gap between $-2 t$ and $E_{0}$ induces a change in the chemical potential, which, in turn, allows for the existence of a finite value of the critical temperature at which spatial BEC occurs. 


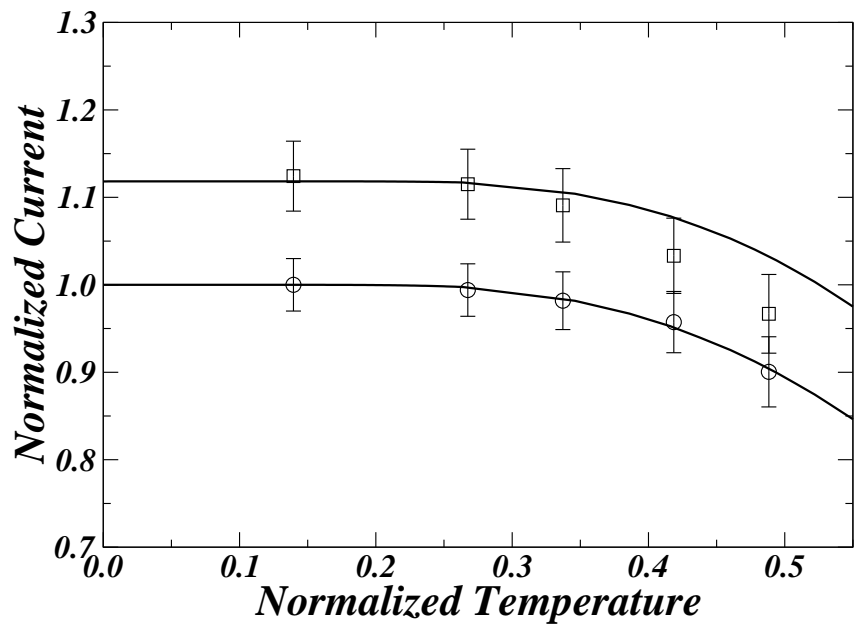

FIG. 5: Critical currents (in units of the critical current on the reference chain at $T=1.2 K$ ) as a function of $T / T_{c}$ for the backbone and the chain. The solid lines are the estimated critical currents for the backbone (top) and the chain (bottom). Circles (squares): experimental values for the chain (backbone).

Fixing the number of particles in the grand canonical partition function amounts to choose the fugacity $z$ so that

$$
N_{T}=\sum_{E \in \sigma} \frac{d(E)}{z^{-1} e^{\beta\left(E-E_{0}\right)}-1} .
$$

In Eq. (57) $N_{T}$ is the number of particles, $d(E)$ is the degeneracy of each single-particle eigenstate, $\beta=1 / k_{B} T$ and $E_{0}$ is the energy of the lowest energy state allowed to a particle hopping on a comb; the sum is taken over the entire spectrum $\sigma$.

For free bosons hopping on a square comb of size $L$ (with $N_{S}=L \times L$ sites), one has

$$
N_{T}=N_{E_{0}}(L, T)+N_{\sigma_{-}}(L, T)+N_{\sigma_{+}}(L, T)+\int_{E \in \sigma_{0}} d E \frac{L^{2} \rho(E)}{z^{-1} e^{\beta(E+\sqrt{8} t)}-1},
$$

where $N_{E_{0}}(L, T), N_{\sigma_{-}}(L, T)$ and $N_{\sigma_{+}}(L, T)$ denote, respectively, the number of particles which, at a certain temperature $T$, occupy the ground-state and the two regions $\sigma_{-}$and $\sigma_{+}$ of the hidden spectrum; $\rho(E)$, with $E \in \sigma_{0}$, is the energy density of states defined in Eq. (40). Upon defining $n_{E_{0}}=N_{E_{0}} / L^{2}, n_{\sigma_{0}}=N_{\sigma_{0}} / L^{2}$ and $n_{\sigma_{ \pm}}=N_{\sigma_{ \pm}} / L^{2}$ as the contribution given by each one of the three spectral regions to the number of particles per site, one has 
- as $L \rightarrow \infty$ - that

$$
\begin{gathered}
n_{E_{0}}(T)=\lim _{L \rightarrow \infty} \frac{1}{N_{S}} \frac{1}{z^{-1}-1}, \\
n_{\sigma_{-}}(T)=\lim _{L \rightarrow \infty} \frac{2}{L^{2}} \sum_{n=1}^{(L-1) / 4} \frac{1}{z^{-1} e^{\beta t\left[\sqrt{8}-2 \sqrt{1+\cos ^{2}(2 \pi n / L)}\right]}-1},
\end{gathered}
$$

and

$$
\begin{aligned}
n_{\sigma_{+}}(T) & =\lim _{L \rightarrow \infty} \frac{1}{L^{2}} \sum_{n=1}^{(L-1) / 4} \frac{2}{z^{-1} e^{\beta t\left[\sqrt{8}+2 \sqrt{1+\cos ^{2}(2 \pi n / L+\pi / 2)}\right]}-1} \\
& <\lim _{L \rightarrow \infty} \frac{2}{L} \frac{1}{z^{-1} e^{\beta t(\sqrt{8}+2)}-1}=0 \quad \forall T .
\end{aligned}
$$

The last equation shows that, at any finite temperature $T, \sigma_{+}$is not macroscopically occupied.

The last term of the right-hand side of Eq. (58) is the number of bosons in the delocalized states. On a chain the integral appearing in Eq. (58) is diverging since the limit $z \rightarrow 1$ is attained when the energy equals $-2 t$; at variance, for a comb, the existence of the hidden spectrum renders the same integral convergent since the limit $z \rightarrow 1$ is now attained at the lower energy $-2 t \sqrt{2}$ which lies outside the interval $-2 t, 2 t$. One then sees explicitly how the network's inhomogeneity works to induce the emergence of a spatial BEC even if the bosons are "free" and the network is one-dimensional.

If one defines as $T_{c}$ the critical temperature at which spatial BEC on the comb's backbone occurs, then, for any $T<T_{c}$, the localized ground-state is macroscopically filled. Since $n_{E_{0}}\left(T_{c}\right)=0$, using Eqs. (40) and (58), $T_{c}$ may be determined (as a function of the filling fraction $f$ and of the hopping strength $t$ ) from

$$
\pi f=\int_{-2 t}^{2 t} \frac{d E}{\sqrt{4 t^{2}-E^{2}}} \frac{1}{e^{\left(E-E_{0}\right) /\left(k_{B} T_{c}\right)}-1} .
$$

Equation (59) can be solved numerically for any value of $f$. When $f \gg 1$, one may expand the exponential in Eq. (59) to the first order in the inverse of the critical temperature $T_{c}$ getting

$$
\frac{\pi f}{k_{B} T_{c}} \approx \int_{-2 t}^{2 t} d E \frac{1}{\sqrt{4 t^{2}-E^{2}}} \frac{1}{E-E_{0}} .
$$

Substituting $\cos \theta=E / 2 t$ in Eq. (60), one has

$$
\frac{2 t \pi f}{k_{B} T_{c}}=\int_{0}^{\pi} \frac{d \theta}{\cos \theta-E_{0} / 2 t},
$$


from which

$$
k_{B} T_{c}=E_{J} \sqrt{\left(\frac{E_{0}}{2 t}\right)^{2}-1},
$$

with $E_{J}$ being the Josephson energy defined in Eq. (27). Equation (61) allows for a simple estimate of the critical temperature $T_{c}$ at which BEC occurs for free bosons hopping on a comb. Upon inserting the pertinent value of the ground-state energy $E_{0}$ in Eq. (60), one finally gets

$$
k_{B} T_{c} \approx E_{J}
$$

A similar analysis can be carried out for the variety of comb-shaped networks depicted in fig. 4 .

The condensate's fraction may be easily determined as a function of the scaled temperature $\tau=\frac{T}{T_{c}}$. Taking into account that

$$
N_{\sigma_{0}}(\tau)=\lim _{L \rightarrow \infty} N_{S} \int_{-2 t}^{2 t} \rho(E) \frac{d E}{e^{\beta\left(E-E_{0}\right)}-1} \approx N_{T} \cdot \tau,
$$

expanding to the first order in $\beta$ the exponential appearing in Eq. (63) [38], and, finally, using Eqs. (58) and (63), one may easily compute the number of particles occupying the states belonging to $\sigma_{-}$. Namely, one has to compute $N_{0}=N_{E_{0}}+N_{\sigma_{-}}$, where $N_{E_{0}}\left(N_{\sigma_{-}}\right)$is the number of particles in the ground state (in $\sigma_{-}$, except for those occupying the ground state): as a result the fraction of condensate, for $T<T_{c}$, is given by

$$
\frac{N_{0}}{N_{T}} \approx 1-\tau
$$

For $f$ ranging from $10^{3}$ to $10^{9}$, the results provided by Eq. (64) differ from those obtained by the numerical evaluation of $N_{0}$ from Eq. (58) by less than 1\%. Equation (64) shows that the condensate has dimension 1 .

Finally, one may compute the average number of bosons $N_{B}(x, y)$ occupying a generic site $(x, y)$ of a comb [32]; of course, this number depends only on $y$ due to the translational invariance of a comb along the backbone. One finds that $N_{B}(y ; \tau)$ is given by:

$$
\begin{aligned}
N_{B}(y ; \tau) & =N_{E_{0}}(\tau)\left|\psi_{E_{0}}(y)\right|^{2}+\sum_{E_{n} \in \sigma_{-}} N_{\sigma_{-}}\left(E_{n} ; \tau\right)\left|\psi_{E_{n}}(y)\right|^{2} \\
& +L^{2} \int_{E \in \sigma_{0}} d E \rho(E) \frac{1}{e^{\beta(E+\sqrt{8} t)}-1}\left|\psi_{E}(y)\right|^{2} .
\end{aligned}
$$

In Eq. (65) $\psi_{E_{0}}(y)$ is the wavefunction corresponding to the ground-state of the singleparticle spectrum and $\psi_{E_{n}}(y)$ are the eigenfunctions corresponding to the energies $E_{n}$ of the 


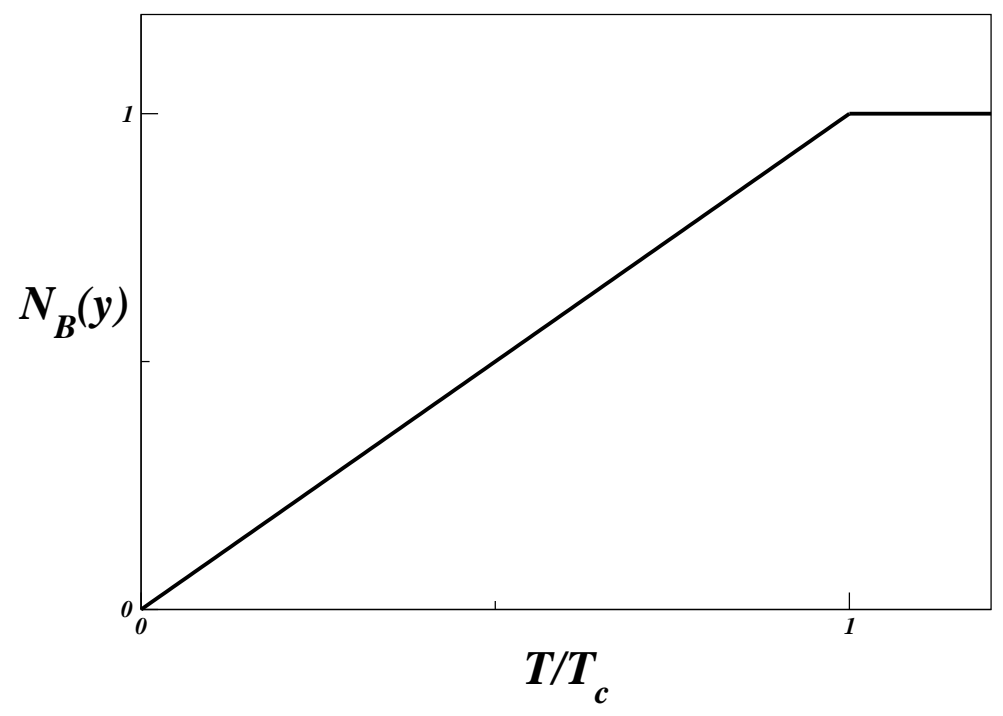

FIG. 6: Distribution of the number of bosons $N_{B}$ as a function of $T / T_{c}$ computed for $y \gg 1$, very far from the backbone. $N_{B}(y)$ is in units of the filling $f$ and is therefore equal to 1 for $T \geq T_{c}$.

hidden spectrum $\sigma_{-} ; N_{\sigma_{-}}\left(E_{n}\right)$ is the number of particles with energies $E_{n} \in \sigma_{-}$and $N_{E_{0}}$ is the number of particles in the ground-state. In the last term of Eq. (65) $\psi_{E}(y)$ are the delocalized eigenfunctions of the eigenvalue equation (29). For determining $N_{B}(y ; \tau)$, one needs to compute $N_{E_{0}}$ and $N_{\sigma_{-}}$, which are evaluated [32] in Appendix B. Using these results, an explicit analytical form for the number of bosons at site $y, N_{B}(y)$, may, then, be derived. The last term in Eq. (65) yields, in fact, the contribution coming from the delocalized states: for a large network ( $L \gg 1)$, and far away from the backbone, this number is independent on the site index $y$ and equals a constant $\left(N_{T} / L^{2}\right) \tau$. Using Eqs. (B)7) and (B8), for $\tau<1$, one finds that the distribution of bosons is, for $y \gg 1$, given by [32]

$$
\frac{N_{B}\left(y ; T / T_{c}\right)}{f} \approx \tau
$$

The signature of the emerging spatial BEC in a system of non-interacting bosons hopping on a comb-shaped network is provided then by the remarkably sharp decrease of the number of bosons at sites located away from the backbone. The linear dependence exhibited by the solid line in Fig. 6 is consistent with the observation that the condensate has dimension 1. 


\section{CONCLUDING REMARKS}

We evidenced how the optimal engineering of the shape of a network leads to the emergence of complex features in quantum devices realized with bosonic and superconducting networks, whose connectivity is described by an adjacency matrix supporting an hidden spectrum. For this purpose we analyzed the paradigmatic case of comb-shaped bosonic and superconducting networks.

For free bosons hopping on a comb, we evidenced how the network's connectivity is responsible for the emergence of a spatial BEC along the comb's backbone and computed the critical temperature where a spatial BEC emerges. We then analyzed the inhomogeneous distribution of the bosons along the comb fingers and estimated the dependence of the noncondensate fraction on the reduced temperature $\tau$; we showed that the signature for the emergence of a spatial BEC on the comb's backbone is provided by a rather sharp decrease of the number of bosons occupying the fingers as the temperature is lowered below $T_{c}$. Finite size corrections to our results are negligible already for $f \sim 100$. With little modifications our analysis could be carried out also for diverse network's topologies supporting BEC [31].

We analyzed also superconducting JJNs fabricated on a comb-shaped insulating support. We showed that a non perturbative (i.e., induced by the states of the hidden spectrum) renormalization of some (i.e., the ones located on the backbone) of the Josephson couplings of a comb-shaped JJN is responsible for the observed enhancement of $I_{c}$ of the Josephson junctions located along the comb's backbone. We used an effective theory based on the $\mathrm{BdG}$ equations since it allows for a simple and rather intuitive derivation of Eq. (53), which evidences the crucial role played by the hidden spectrum in determining the enhancement of the Josephson current along the comb's backbone. The BDG approach relies on a few key assumptions; namely, that the eigenfunctions of the $\mathrm{BdG}$ equations may be written in a tight binding form and that only the electrons close to the Fermi surface contribute to determine $E_{J}$; once these assumptions are made, one is able to derive Eqs. (10)- (11) and to account for all the dependence on the electronic states into the definition of the parameters $E_{J}$ and $\tilde{\mathcal{V}}$, which may be determined [22] from the measurements carried in [21] on the linear chain. Our approach yields a value of the renormalized Josephson coupling of the junctions located on the comb's backbone in excellent agreement with the experimental results (see fig.5). Similar phenomena happen for the class [31] of JJNs fabricated on graphs whose 
adjacency matrix supports an hidden spectrum.

An alternative way to look at comb-shaped networks is to regard them as a linear chain immersed in an environment mimicked by the addition of the fingers. This situation may be analyzed using either the Caldeira-Leggett [39] or - for superconducting devices- the electromagnetic environment [40] models. For Josephson devices this point of view was advocated long ago in [41]. For these devices one expects that the nominal value of the Josephson energy $E_{J}$ of the junctions in the array gets renormalized by the interaction with the environment. However, one usually assumes that the effective boundary conditions for the quantum fluctuations of the environment modes do not depend on the Josephson couplings or on the network's topology: while this assumption may be perfectly legitimate for weak environmental fluctuations, better care should be used if these fluctuations are strong as it may well happen for one dimensional JJNs. A simple paradigmatic example of a non perturbative renormalization of Josephson couplings is given by the simple inhomogeneous one-dimensional array analyzed in [42, 43], where the source of inhomogeneity is given by putting on a site of the linear chain a test junction with a different nominal value of the Josephson coupling $E_{J}$. Our analysis shows that, for a comb-shaped JJN, the Josephson couplings on the backbone get renormalized and that this renormalization is non perturbative since the peculiar connectivity of a comb modifies the spectrum of quantum modes living on linear chains by the (obviously non-perturbative) addition of an infinite set of localized states below the continuum threshold: adding the fingers to a backbone chain is, in fact, a topological operation since it amounts to a non trivial change of boundary conditions for the Josephson linear chain. It would be interesting to investigate in this perspective also systems realized with cold atoms living on pertinent inhomogeneous optical lattices.

Our analysis provides experimentally testable examples of how the space connectivity affects coherent behaviors of physical systems.

\section{Acknowledgements}

We benefited from discussions with M. Cirillo, D. Giuliano, G. Giusiano, A. Montorsi, M. Rasetti, B. Ruggiero, R. Russo, P. Silvestrini. Our research has been partially supported by the MUR Project Josephson Networks for Quantum Coherence and Information (grant No.2004027555). P. S. thanks the the Progetto Lagrange and the Statistical Theory Group 
at S.I.S.S.A. for financial support during his residence at the Politecnico di Torino and S.I.S.S.A..

\section{APPENDIX A: SOLUTION OF LBDG FOR A JJ-CHAIN}

Let us consider a Josephson chain with $N_{S}$ grains: $i=1, \cdots, N_{S}$ and $\alpha \rightarrow k$, with $k=2 \pi n / N_{S}$ and $n=-N_{S} / 2, \cdots, N_{S} / 2-1$ (periodic boundary conditions are used). Since $A_{i j}=\delta_{i, j+1}+\delta_{i, j-1}$, one gets that the eigenvalues of the matrix $\epsilon_{i j}$ are

$$
E_{k}=-2 t \cos k+U_{c}-\mu
$$

where $U(i) \equiv U_{c}$ does not depend on the grain position. Similarly, one sets $\Delta(i) \equiv \Delta_{c}$. One finds then $u_{k}(j)=N_{S}^{-1 / 2} U_{k} e^{i k j}$ and $v_{k}(j)=N_{S}^{-1 / 2} V_{k} e^{i k j}$, which, in turn, lead to $U_{k}^{2}=$ $(1 / 2)\left[1+E_{k} / \epsilon_{k}\right]$ and $V_{k}^{2}=(1 / 2)\left[1-E_{k} / \epsilon_{k}\right]$, where $\epsilon_{k}=\sqrt{\Delta_{c}^{2}+E_{k}^{2}}$. From (10) one gets $1=\left(\tilde{\mathcal{V}} / 2 N_{S}\right) \sum_{k} \in_{k}^{-1} \tanh \left(\beta \in_{k} / 2\right)$ and, for $N_{S} \gg 1$, one finally obtains

$$
1=\frac{\tilde{V}}{4 \pi t} \int_{-2 t}^{2 t} \frac{d E}{\sqrt{1-\frac{E^{2}}{4 t^{2}}} \sqrt{\Delta_{c}^{2}+\left(E-\mu+U_{c}\right)^{2}}} \tanh \left(\frac{\beta}{2} \sqrt{\Delta_{c}^{2}+\left(E-\mu+U_{c}\right)^{2}}\right) .
$$

A BCS-like behavior is obtained with $U_{c}-\mu \approx 0$ : Eq. (A3) for $\Delta_{c}$ reads then

$$
1=\frac{\tilde{V}}{4 \pi t} \int_{-2 t}^{2 t} \frac{d E}{\sqrt{1-\frac{E^{2}}{4 t^{2}}} \sqrt{\Delta_{c}^{2}+E^{2}}} \tanh \left(\frac{\beta}{2} \sqrt{\Delta_{c}^{2}+E^{2}}\right) .
$$

At first sight equation ( $\mathrm{A3}$ ) appears quite different from the corresponding BCS gap equation (9), basically due to the factor $\left(1-E^{2} / 4 t^{2}\right)^{-1 / 2}$ appearing in the integrand and originating from the density of states on the chain. Nevertheless, the behavior is basically BCS. In fact, at $T=0$, one gets

$$
1=\frac{\tilde{V}}{2 \pi t} \cdot \frac{1}{1+\frac{\Delta_{c}^{2}(T=0)}{4 t^{2}}} \cdot K\left(\frac{1}{1+\frac{\Delta_{c}^{2}(T=0)}{\square} 4 t^{2}}\right),
$$

where $K(x)$ is the complete elliptic integral of first kind [45]. If one takes the limit $\Delta_{c} / t \ll 1$ (which is, in a sense, the equivalent of the limit $n(0) V_{B C S} \ll 1$ in the BCS theory) and recalls that, at $T=0, K(x) \approx(1 / 2) \log 16 /(1-x)$ [45], one obtains

$$
\Delta_{c}(T=0) \approx 8 t e^{-2 \pi t / \tilde{\mathcal{V}}}
$$

which is the $1 D$ equivalent on a chain of the well-known BCS expression $\Delta(T=0)=$ $2 \hbar \omega_{D} e^{-1 / n(0) V_{B C S}}$. At $T=T_{c}\left(\Delta_{c}\left(T=T_{c}\right)=0\right)$, one has

$$
1=\frac{\tilde{V}}{2 \pi t} \int_{0}^{1} \frac{d \epsilon}{\epsilon \sqrt{1-\epsilon^{2}}} \tanh \left(\frac{\epsilon t}{2 k_{B} T_{c}}\right)
$$




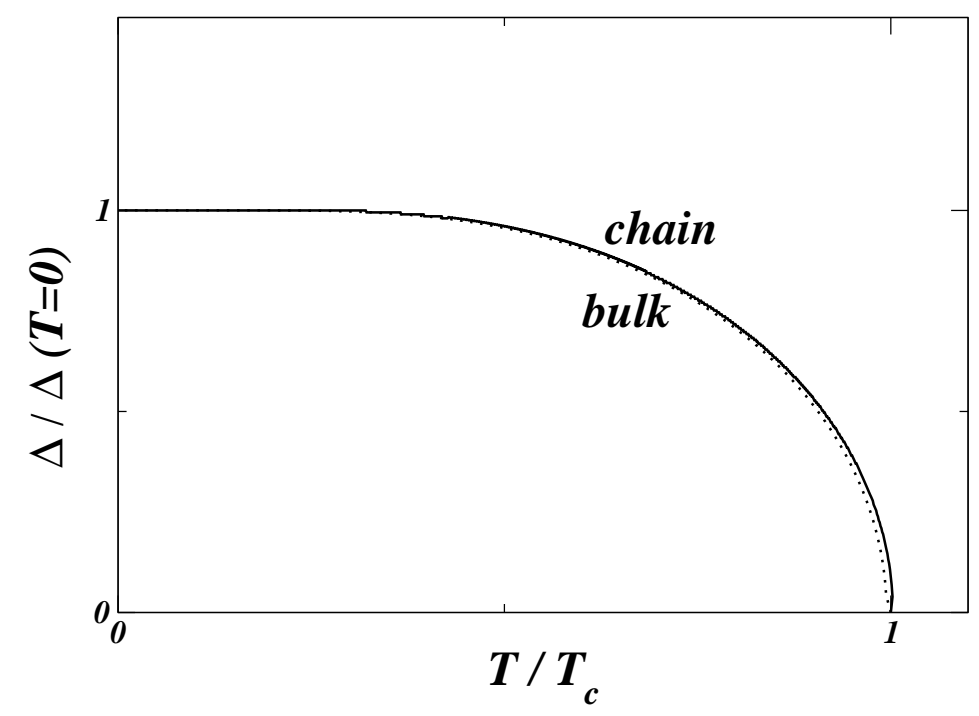

FIG. 7: $\Delta / \Delta(T=0)$ vs. $T / T_{c}$ obtained from the numerical solution of the BCS gap equation (9) with the $N b$ parameters (solid line) and from the numerical solution of the gap equation for the $1 D$ chain of superconducting grains (A3), with the parameters obtained for the setup of [21] (dotted line).

which, for $\Delta_{c} / t \ll 1$, yields $1 \approx(\tilde{V} / 2 \pi t)\left(\log t / k_{B} T_{c}+\log \mathcal{C}\right)$ with $\mathcal{C} \approx 4.536$. As a result one gets

$$
k_{B} T_{c}=\mathcal{C} t e^{-2 \pi t / \tilde{\mathcal{V}}}
$$

which is the $1 D$ equivalent on a chain of the well-known $\mathrm{BCS}$ expression $k_{B} T_{c}=$ $1.14 \hbar \omega_{D} e^{-1 / n(0) V_{B C S}}$. Combining Eqs. (A5) and (A7) enables to show that

$$
\frac{\Delta_{c}(T=0)}{k_{B} T_{c}}=\frac{8}{\mathcal{C}} \approx 1.76
$$

which coincides with the result expected from the BCS theory.

For the experimental setup of [21, 22] one can estimate $t \approx k_{B} \cdot 70 K$ and $\tilde{\mathcal{V}} / t \approx 1.75$, yielding $T_{c} \approx 8.6 K$ and $\Delta_{c}(T=0) \approx k_{B} \cdot 15.9 K$. In Fig. 17, we plot the ratio $\Delta(T) / \Delta(T=0)$ vs. the reduced temperature $T / T_{c}$ obtained from the numerical solution of the BCS gap equation (9) with the $N b$ parameters for the bulk $\left(n(0) V_{B C S}=0.28\right.$ and $\left.\hbar \omega_{D}=k_{B} \cdot 275 K\right)$ and the one obtained from the numerical solution of the gap equation for the $1 D$ chain 
of superconducting grains (A3), with the parameters obtained for the setup of [21]. The comparison evidences the close similarity between the two curves.

Measurements on a chain made with $N b$ grains yield $T_{c} \approx 8.8 K$ and $\Delta_{c}(T=0) \approx$ $1.4 \mathrm{meV} \approx k_{B} \cdot 15.9 \mathrm{~K}$; furthermore, in the experimental setup described in [21, 22] it is $I_{c} \approx 18 \mu A$. The parameters $E_{J}$ and $\tilde{\mathcal{V}}$, determined from Eq. (A7) are then given by $E_{J} \approx$ $k_{B} \cdot 430 K$ and $\tilde{\mathcal{V}} / E_{J}=1.185$.

\section{APPENDIX B: DISTRIBUTION OF BOSONS IN THE HIDDEN SPECTRUM}

In the thermodynamic limit, for $\tau \leq 1, N_{\sigma_{-}}\left(E_{n}, \tau\right)$ is given by [44]

$$
N_{\sigma_{-}}\left(E_{n} ; \tau\right)=\lim _{L \rightarrow \infty} L^{2} \frac{2}{\frac{2 \sqrt{2} t}{k_{B} T}(\pi n)^{2}+\frac{L^{2}}{N_{E_{0}}(\tau)}}
$$

and it depends on the number of particles in the ground-state $N_{E_{0}}$. Since $k_{B} T_{c} \approx 2 t f$, from Eq. (B1), it follows that the number of particles occupying the hidden spectrum is given by

$$
N_{\sigma_{-}}(\tau)=\sum_{n=1}^{\infty} N_{\sigma_{-}}\left(E_{n}\right)=-N_{E_{0}}+N_{E_{0}} \sqrt{\frac{\tau}{\sqrt{2}} \frac{N_{T}}{N_{E_{0}}}} \operatorname{coth}\left[\sqrt{\frac{\tau}{\sqrt{2}} \frac{N_{T}}{N_{E_{0}}}}\right] .
$$

As a consequence of the fact that $N_{0}=N_{\sigma_{-}}+N_{E_{0}}$, from Eq. (64), one finds the way to determine $N_{E_{0}} / N_{T}$ as a function only of the scaled temperature $\tau$ :

$$
N_{T}(1-\tau)=N_{E_{0}} \sqrt{\frac{\tau}{\sqrt{2}} \frac{N_{T}}{N_{E_{0}}}} \operatorname{coth}\left[\sqrt{\frac{\tau}{\sqrt{2}} \frac{N_{T}}{N_{E_{0}}}}\right] .
$$

Solving Eq. (B3) and substituting back the value obtained for $N_{E_{0}}$ in Eq. (B1) allows for an exact numerical evaluation of Eq. (65).

Conventional wisdom supported by numerical evidence suggests that - apart from a small range of temperatures near $T_{c}$ - the largest contribution to $N_{0}$ comes from $N_{E_{0}}$. Thus, it is physically appealing to assume that $N_{E_{0}}$ is given by

$$
N_{E_{0}}=N_{T}(1-\tau) g(\tau)
$$

In Eq. (B4), $g(\tau)$ is a function only of the scaled temperature $\tau$ and parametrizes the contributions to $N_{0}$ coming from the states belonging to the hidden spectrum: when $g=1$, the condensate is in the ground-state, while, for $g=0$, is in the states of the hidden spectrum. 


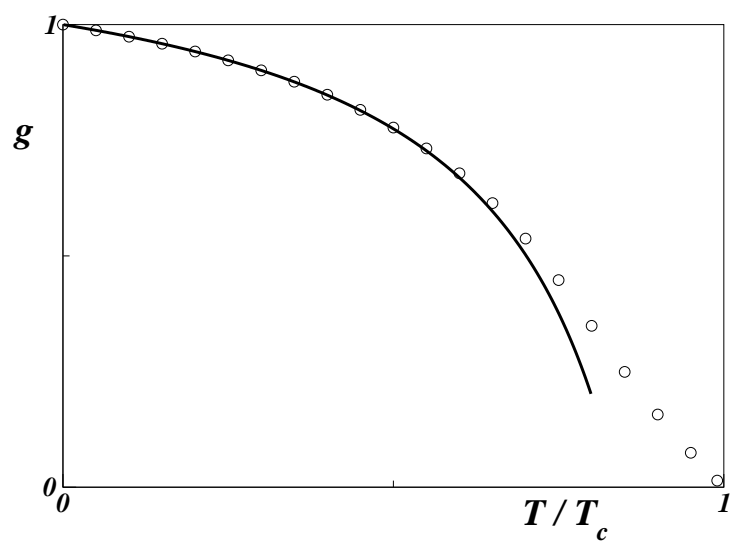

FIG. 8: The function $g(\tau)$ defined in Eq. (Bי $)$ : the empty circles correspond to the numerical solution of the self-consistency equation (B-B5); the solid line corresponds to the approximate expression (B6).

Substituting Eq. (B4) in Eq. (B1) and requiring $N_{0}=N_{\sigma_{-}}+N_{E_{0}}$ with $N_{0}$ given by Eq. (64), leads to a self-consistency equation for $g(\tau)$ :

$$
g(\tau) \sqrt{\frac{\tau}{\sqrt{2}(1-\tau) g(\tau)}} \operatorname{coth}\left[\sqrt{\frac{\tau}{\sqrt{2}(1-\tau) g(\tau)}}\right]=1 .
$$

For $\tau$ not too close to 1, a rather simple approximate solution of Eq. (B5) is provided by

$$
g(\tau) \approx 2-\sqrt{\frac{\tau}{\sqrt{2}(1-\tau)}} \operatorname{coth}\left[\sqrt{\frac{\tau}{\sqrt{2}(1-\tau)}}\right] .
$$

The error made in using Eq. (B6) instead of the exact solution of Eq. (B5) is within few percents: for $\tau \leq 0.5$ the error is less than $1 \%$, while for $\tau=0.7$ is about $5 \%$. In Fig. (8) we plot the function $g(\tau)$ as obtained from the numerical solution of the self-consistency Eq. (B5) and from the approximate expression (B6).

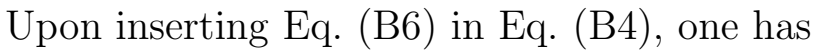

$$
\frac{N_{E_{0}}(\tau)}{N_{T}} \approx(1-\tau)\left(2-\sqrt{\frac{\tau}{\sqrt{2}(1-\tau)}} \operatorname{coth}\left[\sqrt{\frac{\tau}{\sqrt{2}(1-\tau)}}\right]\right),
$$

and, from Eq. (B1), one gets

$$
\frac{N_{\sigma_{-}}(\tau)}{N_{T}}=\sum_{n=1}^{\infty} \frac{2}{\frac{\sqrt{2}(\pi n)^{2}}{\tau}+\frac{1}{(1-\tau) g(\tau)}} \approx(1-\tau)\left\{\sqrt{\frac{\tau}{\sqrt{2}(1-\tau)}} \operatorname{coth}\left[\sqrt{\frac{\tau}{\sqrt{2}(1-\tau)}}\right]-1\right\}
$$

[1] N. Goldenfeld and L. Kadanoff, Science 284, 87 (1999). 
[2] E. Dagotto, Science 309, 257 (2005).

[3] P. Xiong et al., Phys. Rev. Lett. 69, 3220 (1992); E. Fradkin and S. A. Kivelson, Phys. Rev. 59, 8065 (1999); S. A. Kivelson, E. Fradkin and V.J. Emery, Nature 393, 550 (1998).

[4] E. Dagotto, T. Hotta and A. Moreo, Phys. Rep. 344, 1 (2001); E. Dagotto, Nanoscale Phase Separation and Colossal Magnetoresistance, Springer-Verlag (2001).

[5] V.J. Emery, S.A. Kivelson and J.M. Tranquada, Proc. Natl. Acad. Sci. U.S.A. 96, 8814 (1999); J. Zaanen, Nature 404, 714 (2000) and references therein.

[6] Ch. Renner et al., Phys. Rev. Lett. 80, 149 (1998); M. Suzuki, T. Watanabe and A. Matsuda, Phys. Rev. Lett. 82, 5361 (1999); T. Ekino et al. J. Low Temp. Phys. 117, 359 (1999).

[7] S.A. Kivelson and E. Fradkin, Preprint cond-mat/0507459.

[8] Yu.N. Ovchinnikov, S.A. Wolf and V.Z. Kresin, Phys. Rev. B63, 064524 (2001).

[9] P.W. Anderson, Science 177, 393 (1972).

[10] see, for instance: Coherence in Superconducting Networks, J.E. Mooij and G. Schön eds., Physica B152, pp.1-308, (1988); Josephson Junction Arrays, H.A. Cerdeira and S.R. Shenoy eds., Physica B222, pp.253-406, (1996); R. Fazio and H. van der Zant, Phys. Rep. 355, 235 (2001).

[11] O. Morsch and M. K. Oberthaler, Rev. Mod. Phys. 78, 179 (2006); F.S. Cataliotti et al., Science 293, 843 (2001); B.P. Anderson and M. Kasevich, Science, 282, 1686 (1998).

[12] E. Simànek, Inhomogeneous Superconductors, Oxford University Press, New York, 1994.

[13] Y. Makhlin, G. Shoen and A. Shnirman, Rev. Mod. Phys. 73, 357 (2001).

[14] P.G. de Gennes, C. R. Acad. Sci. Ser. B292, 279 (1981); P.G. de Gennes, C. R. Acad. Sci. Ser. B292, 9 (1981); S. Alexander, Phys. Rev. B27, 1541 (1983); H.J. Fink, A. Lopez, and R. Maynard, Phys. Rev. B26, 5237 (1982); R. Rammal, T.C. Lubensky, and G. Toulouse, Phys. Rev. B27, 2820 (1983).

[15] G. Deutscher and R. Rosembaum, Appl. Phys. Lett 27, 366 (1975); G. Deutsher, I. Grave and S. Alexander, Phys. Rev. Lett. 48, 1497 (1982); G. Deutscher et al., Phys. Rev. B24, 6464 (1981).

[16] J. Berger, J. Rubinstein eds. Connectivity and Superconductivity, Lecture Notes in Physics, Springer-Verlag, Berlin, 2000.

[17] L.B. Ioffe et al., Nature 415, 503 (2002); B. Doucot, M.V. Feigel'man and L.B. Ioffe, Phys. Rev. Lett. 90, 107003, (2003); B. Doucot, L.B. Ioffe and J. Vidal, Phys. Rev. B69, 107003 
(2003); B. Doucot et al. Phys. Rev. B 71, 024505 (2005).

[18] X.G. Wen and Q. Niu, Phys. Rev. B41, 9377 (1990); X.G. Wen, Phys. Rev. Lett. 90, 016803 (2003).

[19] C.C. Abilio et al., Phys. Rev. Lett. 83, 5102 (1999); J. Vidal, R. Mosseri and B. Doucot, Phys. Rev. Lett. 81, 5888 (1998).

[20] I.V. Protopopov and M. V. Feigel'man, Phys.Rev. B70, 184519 (2004); I.V. Protopopov and M.V. Feigel'man, cond-mat/0510766.

[21] P. Silvestrini, R. Russo, V. Corato, B. Ruggiero, C. Granata, S. Rombetto, M. Russo, M. Cirillo, A. Trombettoni, and P. Sodano, cond-mat/0512478.

[22] P. Sodano, A. Trombettoni, P. Silvestrini, R. Russo, and B. Ruggiero, New J. Phys., 8, 327 (2006).

[23] L. P. Pitaevskii and S. Stringari, Bose-Einstein Condensation, Oxford University Press, Oxford, 2003.

[24] M. K. Oberthaler and T. Pfau, J. Phys.: Condens. Matter 15, R233 (2003).

[25] R. Roth and K. Burnett, Phys. Rev. A 68, 023604 (2003).

[26] I. Brunelli, G. Giusiano, F. P. Mancini, P. Sodano, and A. Trombettoni, J. Phys. B 37, S275 (2004).

[27] M. Albiez, R. Gati, J. Folling, S. Hunsmann, M. Cristiani, and M. K. Oberthaler, Phys. Rev. Lett. 95, 010402 (2005).

[28] A. Smerzi, S. Fantoni, S. Giovanazzi, and S. R. Shenoy, Phys. Rev. Lett. 79, 4950 (1997).

[29] F. Harary, Graph Theory, Addison-Wesley, Reading (1969).

[30] R. Burioni, D. Cassi, I. Meccoli, M. Rasetti, S. Regina, P. Sodano, and A. Vezzani, Europhys. Lett. 52, 251 (2000).

[31] R. Burioni, D. Cassi, M. Rasetti, P. Sodano, and A. Vezzani, J. Phys. B 34, 4697 (2001).

[32] G. Giusiano, F. P. Mancini, P. Sodano, and A. Trombettoni, Int. J. Mod. Phys. B 18, 691 (2004).

[33] P.G. de Gennes, Superconductivity of Metals and Alloys, Addison-Wesley (1989).

[34] B. Abeles, Phys. Rev. B 15, 2828 (1997).

[35] D. Jaksch, C. Bruder, J. I. Cirac, C. W. Gardiner, and P. Zoller, Phys. Rev. Lett. 81, 3108 (1998).

[36] F. P. Mancini, P. Sodano, and A. Trombettoni, to appear in "Eleventh Training Course in 
the Physics of Correlated Electron Systems and High-Tc Superconductors", Vietri sul Mare, Italy, Oct 2006, cond-mat/0612388.

[37] V. Ambegaokar and A. Baratoff, Phys. Rev. Lett. 10, 486 (1963); ibid. 11, 104 (1963)

[38] This approximation holds for $f \gg 1$ and it is in very good agreement with the results of a numerical evaluation of the integral (63).

[39] A.O. Caldeira and A.J. Leggett, Ann.Phys. (N.Y.) 149, 374 (1983).

[40] M.H. Devoret et al., Phys. Rev.Lett. 64, 1824 (1990); S.M. Girvin et al., 64, 3183 (1990); G. Schön and A.D. Zaikin, Phys. Rep. 198, 237 (1990).

[41] A. Schmid, J. Low Temp. Phys. 49, 609 (1982).

[42] L.I. Glazman and A.I. Larkin, Phys. Rev. Lett. 79, 3736 (1997).

[43] D. Giuliano and P. Sodano, Nucl. Phys. B711, 480 (2005).

[44] P. Buonsante, R. Burioni, D. Cassi, and A. Vezzani, Phys. Rev. B 66, 094207 (2002).

[45] M. Abramowitz and I. A. Stegun, Handbook of mathematical functions with formulas, graphs, and mathematical tables, National Bureau of Standards, Washington, D.C., 1964. 